\title{
Auto-antibody evaluation in idiopathic interstitial pneumonia and worse survival of patients with Ro52/TRIM21auto-antibody
}

\author{
Hiroko Morita, ${ }^{1}$ Yasuo Shimizu, ${ }^{1, *}$ Yusuke Nakamura, ${ }^{1}$ Hiroaki Okutomi, ${ }^{1}$ Taiji Watanabe, ${ }^{1}$ Tatsuya Yokoyama, ${ }^{1}$ \\ Sayo Soda, ${ }^{1}$ Naoya Ikeda, ${ }^{1}$ Taichi Shiobara, ${ }^{1}$ Masaaki Miyoshi, ${ }^{1}$ Kazuyuki Chibana, ${ }^{1}$ Akihiro Takemasa, ${ }^{1}$ and \\ Kazuhiro Kurasawa² \\ 'Department of Pulmonary Medicine and Clinical Immunology and ${ }^{2}$ Department of Rheumatology, Dokkyo Medical University School of Medicine, \\ 880 Kitakobayashi, Mibu, Tochigi 321-0293, Japan
}

(Received 18 January, 2020; Accepted 26 February, 2020; Published online 15 May, 2020)

\begin{abstract}
Some patients with interstitial pneumonia (IP) have auto-antibodies, but do not fit the criteria for specific connective tissue diseases. Examination of auto-antibodies is recommended for diagnosis idiopathic pulmonary fibrosis. A prospective cohort study was performed in 285 patients with IP. Eleven auto-antibodies were assessed and patients were followed for 2 years. All 285 patients underwent the myositis panel test (MPT) for 11 auto-antibodies. Among them, $23.5 \%(67 / 285)$ of the patients had a positive MPT and $14.7 \%(42 / 285)$ had connective tissue diseases. Among the 49 MPT positive patients without connective tissue diseases, 29 patients $(59.2 \%)$ were positive for Ro52, including 17 patients with Ro52 mono-positivity. Among interstitial pneumonia patients without connective tissue diseases, the Ro52 mono-positive patients showed worse at 2-years survival than those who were Ro52 negative $(p=0.022, \mathrm{HR}=5.88,95 \% \mathrm{Cl} 1.29-26.75)$. Most of the Ro52 positive patients also showed a low titer of anti-nucleolar antibody. About $20 \%$ of IP patients had auto-antibodies detectable by the MPT, and Ro52 positive patients accounted for more than half of the MPT positive patients without connective tissue diseases. Detection of Ro52 auto-antibodies may be useful for assessing the risk of progression in idiopathic interstitial pneumonia patients without connective tissue diseases and a low anti-nucleolar antibody titer.
\end{abstract}

Key Words: Ro52, TRIM21, interstitial pneumonia, myositis panel, auto-antibody

$\mathrm{M}$ any diseases are associated with interstitial pneumonia (IP) and the causes of this condition are varied. ${ }^{(1,2)}$ Idiopathic interstitial pneumonia (IIP) is defined as a subset of the group of subacute and chronic lung disorders collectively referred to as IP diseases or diffuse parenchymal lung diseases of unknown aetiology. ${ }^{(1,3)}$ Diagnosis of IIP is confirmed by performing appropriate clinical, radiological, and surgical examinations to exclude secondary IP. Some IP patients have autoimmune features that do not fit the criteria for diagnosis of any specific connective tissue disease (CTD), so IIP is diagnosed. ${ }^{(4)}$ It has been proposed that IP with autoimmune features should be classified as undifferentiated connective tissue diseases (UCTD),$^{(5,6)}$ lung-dominant CTD, ${ }^{(7)}$ autoimmune-featured interstitial lung disease (AIF-ILD), ${ }^{(8)}$ or IP with autoimmune features (IPAF). ${ }^{(9)}$

Serological tests for auto-antibodies are often helpful during initial evaluation of IP, ${ }^{(10-12)}$ and auto-antibody positivity is one of the proposed criteria for IP with autoimmune features. Although various novel auto-antibodies have been discovered, it is not always possible to measure these in the clinical setting, ${ }^{(13,14)}$ but a commercial assay recently became available for some new autoantibodies. ${ }^{(15,16)}$ Measuring these auto-antibodies in patients who have IP with autoimmune features could clarify differences between IIPs and CTD-IP, and could contribute to improving the proposed criteria for IP with autoimmune features. In addition, serological tests such as the myositis panel have been recommended for making a diagnosis of idiopathic pulmonary fibrosis in an official American Thoracic Society, European Respiratory Society, Japanese Respiratory Society, and Latin American Thoracic Society clinical practice guideline 2018 . $^{(17)}$

In the present study, immunoassay for auto-antibodies was performed in IP patients by using a myositis panel test (MPT) kit, which provides quantitative serum levels of IgG auto-antibodies targeting the following 11 antigens: Mi-2 $\beta$ (helicase protein, part of the NuRD complex), Ku (thyroid autoantigen), PM-scl100, PM-scl75, Jo-1 (histidyl-tRNA synthetase), SRP (signal recognition particle, ribonucleoprotein complex), PL-7 (threonyl-tRNA synthetase), PL-12 (alanyl-tRNA synthetase), EJ (glycyl-tRNA synthetase), OJ (isoleucil-tRNA synthetase), and Ro52 (tripartite motif 21, TRIM21). ${ }^{(15)}$ Anti-aminoacyl-tRNA synthase (ARS), including anti-Jo-1, anti-PL-7, anti-PL-12, anti-EJ, anti-KS, and anti-OJ antibodies, can be measured with other commercial assays, but not anti-Mi-2 $\beta,-\mathrm{Ku},-\mathrm{PM}-\mathrm{Scl} 75,-\mathrm{SRP}$, and -Ro52 antibodies. Among these antigen, Ro52 ubiquitilates p62/ sequestosome1 (SQSTM1) and supresses protein sequestration to regulate redox homeostasis, and previously we reported that p62/SQSTM1 was co-localized to iron binding silica with oxidative stress lung in mice. ${ }^{(18,19)}$

The present study was performed to clarify the positive rates of 11 different auto-antibodies in IP patients. In addition, after excluding IP patients who met the criteria for diagnosis of CTDs, survival of the remaining anti-Ro52 positive IP patients was investigated.

\section{Methods}

Subjects. A total of 285 patients with a diagnosis of IP based on chest computed tomography (CT) were enrolled. Patients were excluded if they were under 20 years old. CTDs were diagnosed by department of Rheumatology according to the reported criteria, ${ }^{(2-27)}$ and patients in whom CTDs were diagnosed were excluded from further analysis. IP was evaluated according to the Official American Thoracic Society/European Respiratory Society

*To whom correspondence should be addressed.

E-mail: yasuo-s@dokkyomed.ac.jp 
Statement: Update of the International Multidisciplinary Classification of the Idiopathic Interstitial Pneumonias, ${ }^{(3)}$ with chest CT scans being reviewed by three experienced chest physicians who were blinded to symptoms and laboratory test results. Progression of IP or respiratory failure to death was defined as lung-related death. This study was carried out in accordance with the Declaration of Helsinki and informed consent was obtained from the patients. The study was approved by the Human Research Committee of Dokkyo Medical University (no. 2114 and R-9-2), and was registered with the University Hospital Medical Information Network (UMIN 32926).

Blood sampling and analysis. Serum obtained from a peripheral blood sample was stored at $-80^{\circ} \mathrm{C}$ until analysis. Measurement of the anti-nuclear antibody (ANA) titer was carried out by the fluorescent antibody technique (FA), and a titer $\leq 40$ was defined as negative for ANA according to the manufacturer's directions (SRL, Inc., Hachioji, Tokyo, Japan). Antibodies for Mi-2ß, Ku, PM-scl100, PM-scl75, Jo-1, SRP, PL-7, PL-12, EJ, $\mathrm{OJ}$, and Ro52 were detected by using a line immunoassay [EUROLINE Myositis Antigen Profile 3 (IgG) test (MPT); EUROIMMUN, Lubeck, Germany], and a positive result was defined as $2+$ according to the manufacturer's information.

Statistical analysis. Differences between two groups were calculated by using the chi-square test. For comparison of continuous variables, the Mann-Whitney $U$ test was used. Kaplan-Meier analysis was employed to compare survival between groups and the odds ratio (OR) was calculated with the $95 \%$ confidence interval (CI). $P$ value $<0.05$ was considered significant, and $p<0.1$ indicated a trend.

\section{Results}

An outline of the study and the number of patients enrolled are shown in Fig. 1. A total of 285 patients underwent the MPT, with 67 patients $(23.5 \%)$ being positive and 218 patients $(76.5 \%)$ being negative. Characteristics of all enrolled patients are summarized in Supplemental Table 1*. The gender ratio and smoking status were significantly different between MPT positive and MPT negative patients $(p<0.05)$. In both MPT positive and negative patients, chest CT showed fibrotic-nonspecific IP (f-NSIP) and usual IP (UIP) more often than other patterns, but OP was also frequently observed in MPT negative patients.
After extensive workup to detect CTDs, 18 MPT positive patients and 24 MPT negative patients were diagnosed as having CTDs. After excluding these CTD patients, 49 MPT positive and 194 MPT negative patients underwent further analysis (Fig. 1, Table 1). The characteristics of the MPT positive patients (49/ 243, 20.2\%) and MPT negative patients (194/243, 79.8\%) were similar to those before excluding the CTD patients (Supplemental Table $1 *$ and Table 1$)$.

Based on the MPT, patients were divided into three groups: Ro52 mono-positive (Ro52 mono), positive for Ro52 and other antibodies (Ro52 overlap), and Ro52 negative but positive for

Table 1. Characteristics of patients without connective tissue diseases

\begin{tabular}{lcc}
\hline Parameters & MPT positive & MPT negative \\
\hline Total $(n)$ & $49(20.2 \%)$ & $194(79.8 \%)$ \\
\hline Gender $(n)$ & 28 & 125 \\
Male & $21^{*}$ & 69 \\
Female & $70.3(10.7)$ & $70.2(11.0)$ \\
\hline Age [mean (SD)] & & \\
Smoking status $(n)$ & 25 & 118 \\
Former, Current & $24 *$ & 76 \\
Never & $29.3(42.1)$ & $25.7(37.4)$ \\
Pack-year [mean (SD)] & & 63 \\
\hline HRCT pattern $(n)$ & 13 & 75 \\
UIP & 22 & 12 \\
f-NSIP & 8 & 8 \\
C-NSIP & 3 & 27 \\
AIP & 0 & 4 \\
COP & 0 & 2 \\
PPFE & 0 & 3 \\
RB-ILD & 0 & \\
HP & $a n d n u m b r$ &
\end{tabular}

Data are presented as mean \pm SD and number of patients as $n$. MPT, myositis panel test. HRCT pattern are indicated usual interstitial pneumonia (UIP), fibrotic non-specific interstitial pneumonia (f-NSIP), cellular non-specific interstitial pneumonia (c-NSIP), acute interstitial pneumonia (AIP), cryptogenic organizing pneumonia (COP), pleuroparenchimal fibroelastosis (PPFE), respiratory bronchiolitis-associated interstitial lung disease (RB-ILD), and hypersensitivity pneumonia (HP). ${ }^{*} p<0.05$.

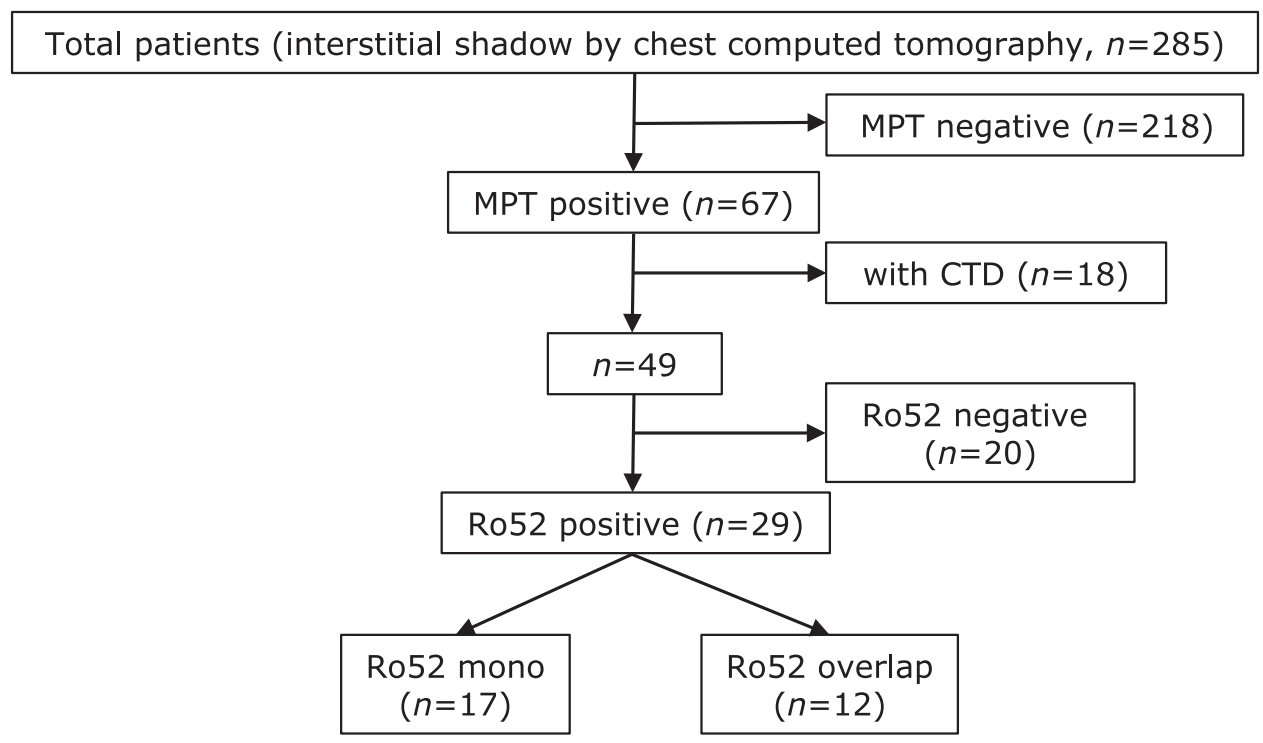

Fig. 1. Study protocol and number of patients enrolled. MPT, myositis panel test; CTDs, connective tissue diseases. Bold arrows indicate main flow of this study. 
Table 2. Clinical symptoms of patients in myositis panel test positive patients

\begin{tabular}{lccc}
\hline $\begin{array}{l}\text { Clinical symptoms related to } \\
\text { connective tissue diseases }\end{array}$ & $\begin{array}{c}\text { Ro52 mono group } \\
(n=17)\end{array}$ & $\begin{array}{c}\text { Ro52 overlap group } \\
(n=12)\end{array}$ & $\begin{array}{c}\text { Ro52 negative group } \\
(n=20)\end{array}$ \\
\hline Symptoms positive & 3 & 2 & 5 \\
Edema of extremities & 3 & 0 & 1 \\
Dry mouth & 0 & 0 & 0 \\
Muscle weakness & 1 & 0 & 1 \\
Mechanic hand & 1 & 0 & 0 \\
Pleural effusion & 0 & 0 & 0 \\
Raynaud & 0 & 0 & 0 \\
Pericardial effusion & 0 & 0 & 0 \\
Fever & 0 & 0 & 1 \\
Neck pain & 0 & 1 & 0 \\
Erythema & 0 & 0 & 2 \\
Dry eye & 0 & 1 & 0 \\
Multiple joint edema & 0 & 0 & 0 \\
Shoulder poikiloderma & 0 & 0 & 0 \\
Joint stiffness & 0 & 0 & 3 \\
Myalgia & 0 & 1 & 0 \\
Symptoms negative & 14 & 10 & 15 \\
\hline Number of patients are presented & Ro52 mono & & \\
\hline
\end{tabular}

Number of patients are presented as $n$. Ro52 mono, Ro52 mono positive in myositis panel test (MPT) patients; Ro52 overlap, Ro52 positive and other antibodies overlap in MPT; Ro52 negative, Ro52 negative but other antibody positive in MPT.

(n)

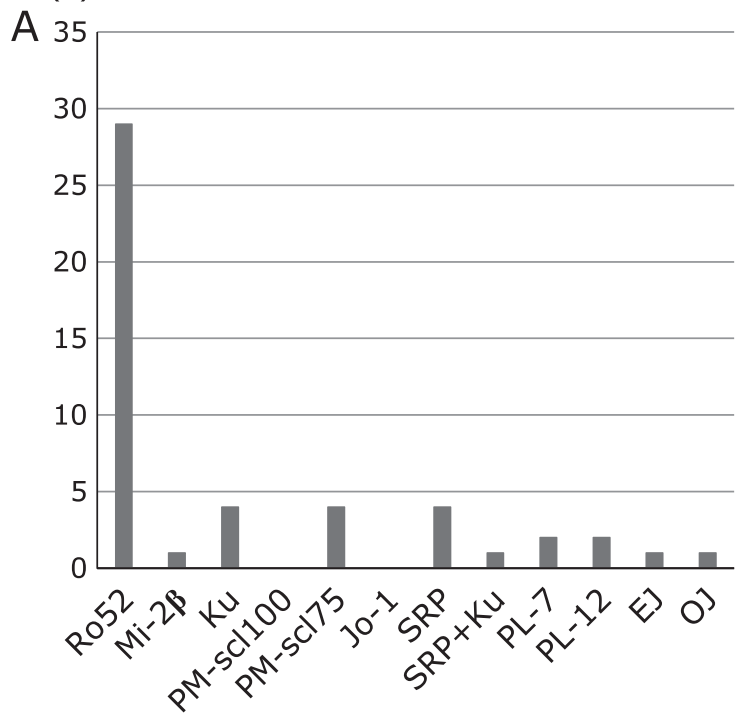

(n)

B 18

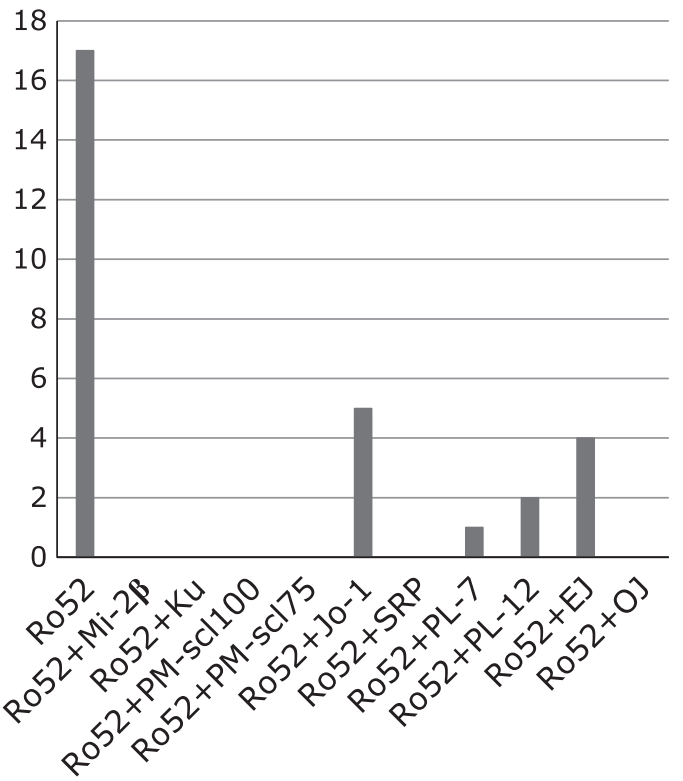

Fig. 2. (A) Number of patients positive for auto-antibodies in the myositis panel test (MPT). MPT positive IP patients without CTDs were investigated $(n=49)$. (B) Number of patients with Ro52 mono-positivity and Ro52 overlap in the MPT. Ro52 positive IP patients without CTDs were investigated $(n=29)$.

other antibodies (Ro52 negative) (Fig. 1). In all 3 groups, most patients had no symptoms related to CTDs, but several patients presented with CTD-like symptoms (Table 2). Edema of the extremities was most often observed in the Ro52 mono group.

Of the 49 MPT positive patients, 29 patients $(29 / 49,59.2 \%)$ were Ro52 positive (Ro52 mono and Ro52 overlap) and 20 patients were Ro52 negative (Fig. 2A). Among Ro52 negative patients, antibodies for $\mathrm{Ku}$, scl-PM75, and SRP were relatively frequent compared to other antibodies. Of the 29 Ro52 positive patients, 17 patients were in the Ro52 mono group (17/29, 58.6\%) and 12 were in the Ro52 overlap group (12/29, 41.4\%) (Fig. 2B).
In the Ro52 overlap group, positivity for Ro52 + Jo-1 and Ro52 + EJ was relatively frequent.

Lung-related death occurred in 8/29 (27.6\%) Ro52 positive patients and 1/20 (5\%) Ro52 negative patients, with lung-related death showing a significantly higher frequency among Ro52 positive patients than Ro52 negative patients ( $p=0.045)$ (Fig. 3A). Among Ro52 positive patients $(n=29)$, lung-related death occurred in $6 / 17(35.3 \%)$ patients in the Ro52 mono group $(n=17)$, as well as in $1 / 5(20.0 \%)$ Ro52 + Jo- 1 patients and $1 / 4(25 \%)$ Ro52 + EJ patients, but in none of the other overlap patients $(n=3)$ (Fig. 3B). The lung-related death rate was not statistically 
$(n)$

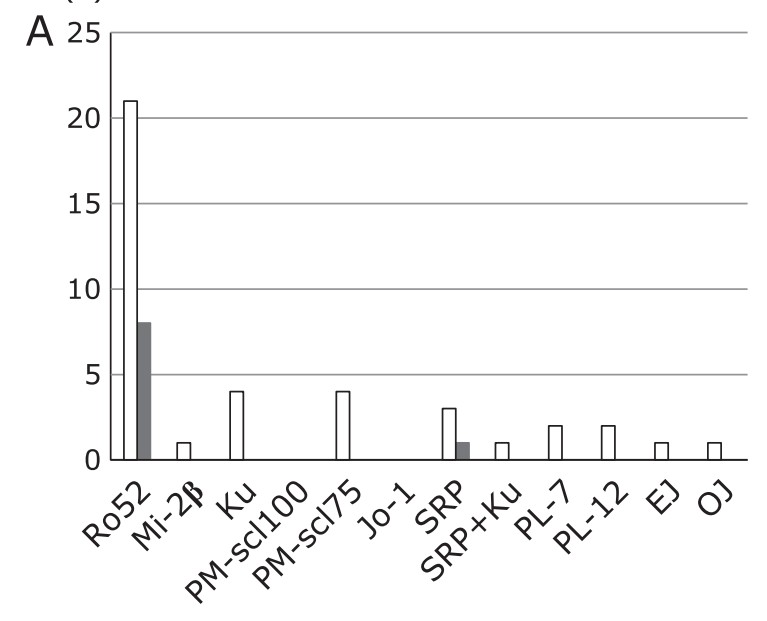

$(n)$

B

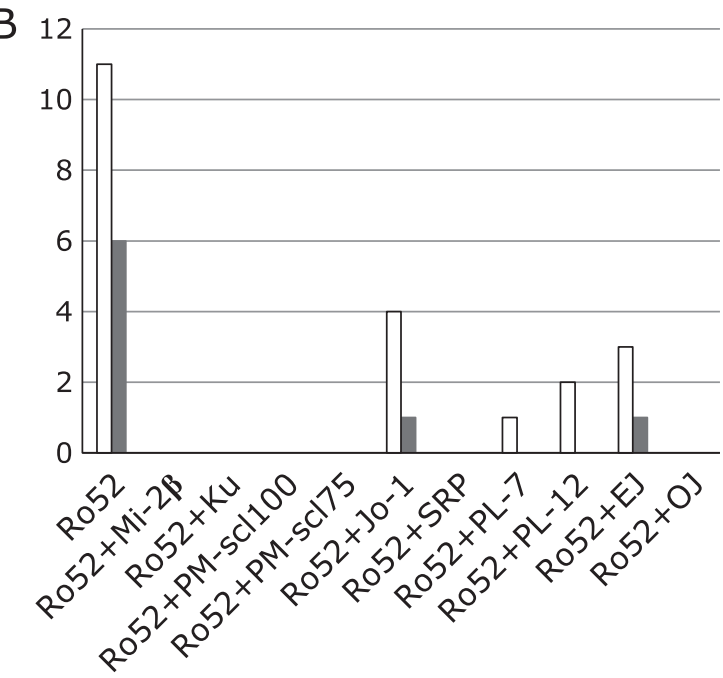

Fig. 3. (A) Positivity for auto-antibodies and lung-related death in the myositis panel test (MPT). MPT positive IP patients without CTDs were investigated $(n=49)$. The observation period was 24 months. (B) Lung-related death among Ro52 positive patients. Ro52 positive IP patients without CTDs were investigated $(n=29)$. The observation period was 24 months.

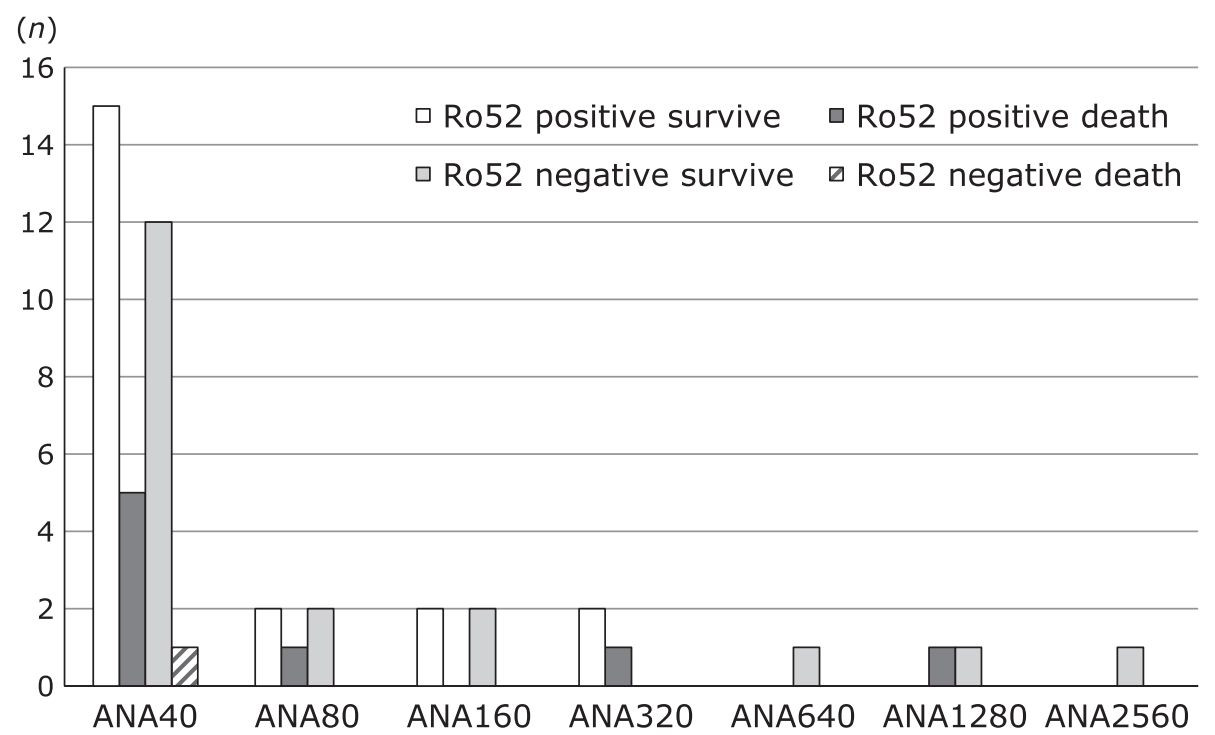

Fig. 4. Lung-related death among patients positive for auto-antibodies in the myositis panel test (MPT) stratified by the ANA titer. MPT-positive IP patients without CTDs were investigated $(n=49)$. The observation period was 24 months. White: surviving Ro52 positive patients, black: Ro52 positive patients who died, mesh: Ro52 negative patients who were positive for other antibodies and survived, diagonal lines: Ro52 negative patients who were positive for other antibodies and died.

different between the Ro52 mono and Ro52 overlap groups. Analysis of lung-related death stratified by the ANA titer was done in Ro52 positive and negative patients (Fig. 4). When ANA was $\leq 40$, lung-related death occurred in $5 / 20(25 \%)$ Ro52 positive patients vs $1 / 13(7.7 \%)$ Ro52 negative patients. When ANA was $\geq 80$, lung-related death occurred in $3 / 9(33.3 \%)$ Ro52 positive patients vs $0 / 7(0 \%)$ Ro52 negative patients. There was no significant difference of lung-related death between Ro52 positive and negative patients with ANA $\leq 40$, or between Ro52 positive patients with ANA $\leq 40$ or ANA $\geq 80$. Analysis of lung-related death stratified by the ANA titer was also done in Ro52 positive patients, comprising the Ro52 mono and Ro52 overlap groups, but there was no significant difference between these two groups (data not shown).

The background of MPT positive patients with lung-related death $(n=8)$ is shown in Table 3 . Six of the eight patients were in the Ro52 mono group. The number of male and female patients was similar. On chest CT, f-NSIP was the most frequent pattern and UIP was next, but some patients showed cellular-NSIP (c-NSIP) and acute IP (AIP). Most patients had a low ANA titer, although two patients showed a high titer. Six patients presented with acute exacerbation and two patients had chronic progressive IP.

When 2-year survival was compared between Ro52 positive and negative patients, the Ro52 positive patients had an increased risk of death relative to Ro52 negative patients $(p=0.0518$, $\mathrm{HR}=3.78$, 95\% CI 0.99-14.45) (Supplemental Fig. $1 *$ ). Next, 
Table 3. Characteristics of patients of lung related death in myositis test positive

\begin{tabular}{lcccc}
\hline Patient & M/F & CT & ANA titer & $\begin{array}{c}\text { Clinical symptoms related to } \\
\text { connective tissue disease }\end{array}$ \\
\hline $\begin{array}{l}\text { Ro52 mono } \\
\text { Pt1 }\end{array}$ & M & UIP & 320 (speckled) & $\begin{array}{c}\text { None } \\
\text { Muscle weakness, erythema } \\
\text { of PIP with edema }\end{array}$ \\
Pt2 & M & f-NSIP & None \\
Pt3 & M & f-NSIP & 1,280 (speckled) & None \\
Pt4 & F & C-NSIP & $\leq 40$ & None \\
Pt5 & F & AIP & 80 & None \\
Pt6 & F & f-NSIP & $\leq 40$ & None \\
\hline Ro52 overlap & M & UIP & $\leq 40$ & None \\
pt7 (Ro52 + EJ) & M & UIP & $\leq 40$ & \\
pt8 (Ro52 + Jo1) & M &
\end{tabular}

$\mathrm{Pt}$, patient; M/F, male/female; ANA, antinuclear antigen; PIP, proximal interphalangeal joint.

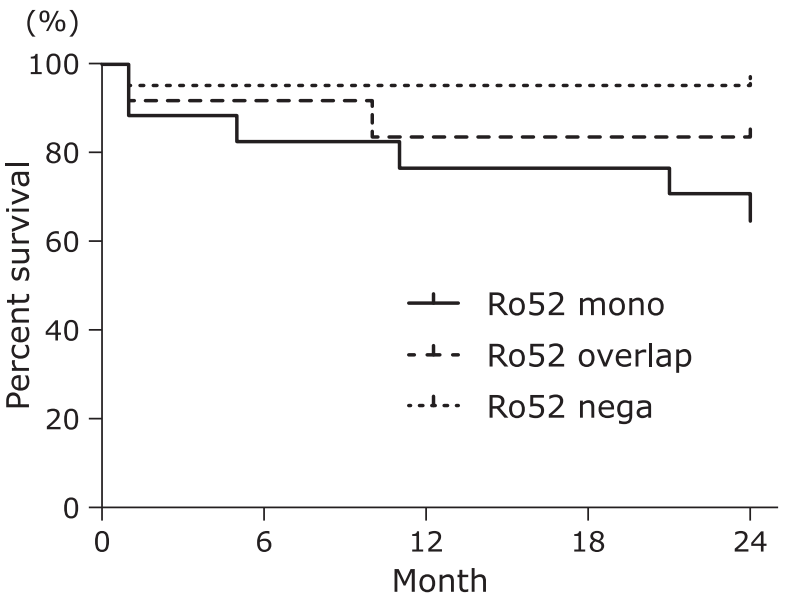

Fig. 5. Kaplan-Meier curves comparing survival of the Ro52 monopositive group (Ro52mono, $n=17$ ), Ro52 overlap group (Ro52overlap, $n=12$ ), and Ro52 negative group (Ro52nega, $n=20$ ) up to 24 months. IP patients without CTDs who were positive in the myositis panel test (MPT) were investigated $(n=49)$.

2-year survival was compared among the Ro52 mono, Ro52 overlap, and Ro52 negative groups. The Ro52 mono group showed significantly worse survival than the Ro52 negative group $(p=0.022, \mathrm{HR}=5.88,95 \%$ CI $1.29-26.75)$, but there was no statistical difference compared with the Ro52 overlap group (Fig. 5). The ratio for the patients receiving immunosuppressive agents were not different among the groups.

\section{Discussion}

This study revealed that anti-Ro52 monospecific positive patients without CTDs had worse 2-year survival than Ro52 negative patients, even though most of them showed a low ANA titer.

CTDs are a major cause of IP in the USA, accounting for about $20 \%$ of patients, while a multi-ethnic study performed in Paris showed that CTDs occurred in about $16 \%$ of IP patients. ${ }^{(28,29)}$ In present study, CTDs were found in $14.7 \%(42 / 285)$ of IP patients. Since this study was performed to investigate patients with serological autoimmune features that did not fulfill the criteria for established CTDs, the 42 CTD patients were excluded from analysis (their characteristics are shown in Supplemental Table 2*).

In the absence of defined CTDs, 10 to $20 \%$ of IIPs patients have been reported to show serological abnormalities. ${ }^{(5)}$ After excluding IP patients with CTDs from the study population,
$20.2 \%(49 / 243)$ of the remaining IP patients were MPT positive. A previous investigation of the prevalence of auto-antibodies revealed MPT positivity in a high $37.5 \%$ (12/32) of IIP patients. ${ }^{(30)}$ Possible reasons for the difference from the present study include a high ANA positive rate of $66.7 \%(8 / 12)$ among MPT positive patients in the other study and its small patient population. ${ }^{(30)}$ Another investigation of these antibodies showed that anti-Ro52 was not only frequently detected in idiopathic inflammatory myositis, but was also prevalent in other CTDs. ${ }^{(31)}$ In patients with systemic sclerosis ( $\mathrm{SSc}$ ), Ro52 positivity was associated with concomitant IP and with worse survival. ${ }^{(32)}$ However, the prognosis of patients with IIPs and Ro52 auto-antibody positivity has not been investigated.

Among the 11 auto-antibodies investigated in this study, Ro52 was detected most frequently. Among the Ro52 positive patients, patients with Ro52 mono-positivity were more frequent $(35.3 \%)$ than those with other auto-antibodies. According to the literature, the prevalence of Ro52 mono-positive patients with IP accompanied by CTDs is $50 \%$ among patients with mixed connective tissue disease (MCTD) ${ }^{(33)} 80 \%$ among those with idiopathic inflammatory myopathies, ${ }^{(31)} 57 \%$ or $36.2 \%$ among those with $\mathrm{SSc},{ }^{(32,34)}$ and $5.6 \%$ among those with UCTD. ${ }^{(35)}$ Healthy subjects were not positive for Ro52 in another study. ${ }^{(13)}$ Based on these reports, the frequency of Ro52 mono-positivity seems to be lower among non-CTD patients with IP than among patients with established CTDs and IP, but higher than among patients with UCTD and IP.

In Ro52 positive patients with SSc, symptoms other than IP showing a higher frequency than in Ro52 negative patients were reported to be fecal incontinence, hyperalimentation, gastroesophageal symptoms, and pulmonary hypertension. ${ }^{(30)}$ In the present study, edema of the extremities was most frequent in the Ro52 mono and Ro52 negative groups. This result might have been obtained because edema was easy to detect during examination and because Ro52 positivity reflects systemic edema. ${ }^{(36,37)}$ It was reported that Ro52 positivity is not specific to myositis and is more prevalent in other CTDs. ${ }^{(16)}$ This could be another reason for the lack of specific symptoms other than IP among Ro52 positive patients.

There have been no reports about the chest CT features of IIP on MPT positive or Ro52 positive patients. Although it was reported that among Norwegian patients with MCTD, Ro52 mono-positive patients more frequently showed severe pulmonary fibrosis than Ro52 negative patients, the detailed CT findings were not described. ${ }^{(33)}$ Among seven IPAF patients who developed acute respiratory distress syndrome (ARDS), it was reported that six patients were Ro52 positive. Of these six patients, CT scans obtained before the onset of ARDS revealed OP in four, acute IP/diffuse alveolar damage (AIP/DAD) in one, and lymphatic interstitial pneumonia (LIP) in one. ${ }^{(38)}$ In present study, f-NSIP 
was the most frequent pattern and UIP was next, but some patients showed cellular-NSIP (c-NSIP) and acute IP (AIP). Patients with OP and c-NSIP are considered to show a better response to corticosteroids or immunosuppressive agents than f-NSIP and UIP. ${ }^{(39)}$ However, if patients are Ro52 positive, it seems important to be aware of the possibility of a poor response to treatment and the elevated risk of lung-related death. A previous study of SSc revealed that Ro52 positive patients with IP showed worse survival after 40 months, especially Ro52 mono-positive patients. ${ }^{(32)}$ In the present study, there was no statistical difference of survival between the Ro52 mono and Ro52 overlap groups up to 2 years, but the Ro52 mono group had significantly worse survival than the Ro52 negative group. The reason for this difference of survival between Ro52 mono-positive patients and Ro52 overlap patients is unknown. The number of patients studied might have affected the results obtained or co-existence of other auto-antibodies could influence the disease phenotype. ${ }^{(32,40,41)}$

There have been no investigations of the association between lung-related death and the ANA titer in non-CTD Ro52 positive or negative patients with IP. The number of lung-related deaths was highest among Ro52 positive patients with ANA $\leq 40$, but the lung-related death rate showed no statistical difference in relation to the ANA titer. Ro52 is abundant in the cytoplasm, and this could lead to a negative result for ANA by fluorescent antibody testing in Ro52 positive patients. Based on our findings, it is important to carefully manage non-CTD Ro52 positive patients with IP and any ANA titer, and those who are ANA negative but Ro52 positive also have a risk of developing severe IP. In the present study, a patient who was anti-SRP positive but Ro52 negative also developed IP and died. It was reported that anti-SRP positive patients were less likely to be complicated by IP than patients with other myositis-related auto-antibodies, ${ }^{(31,42)}$ but some of these patients developed severe IP in other studies. ${ }^{(43,44)}$

Inhibition of Ro52 signaling has been proposed to be involved in induction of IL-17 production and inflammation. ${ }^{(45)}$ Ro52 possesses RING-dependent E3-ubiquitine ligase activity and negatively regulates the production of pro-inflammatory cytokines, and also plays an essential role in p62/SQSTM1-regulated redox homeostasis. ${ }^{(18,45)}$ In addition, binding of IgG Fc to the PRYSPRY domain allows Ro52 to act as a cytoplasmic Fc antibody receptor. ${ }^{(46-48)}$ In mice, Ro52 knockout induces tissue inflammation and systemic autoimmunity via upregulation of the IL-23/IL17 pathway. ${ }^{(37)}$ In patients with inflammatory bowel disease, expression of Ro52 is reduced in CD4+ T cells infiltrating the

\section{References}

1 American Thoracic Society/European Respiratory Society international multidisciplinary consensus classification of the idiopathic interstitial pneumonias. Am J Respir Crit Care Med 2002; 165: 277-304.

2 Wuyts WA, Cavazza A, Rossi G, Bonella F, Sverzellati N, Spagnolo P. Differential diagnosis of usual interstitial pneumonia: when is it truly idiopathic? Eur Respir Rev 2014; 23: 308-319.

3 Travis WD, Costabel U, Hansell DM, et al. An official American Thoracic Society/European Respiratory Society statement: update of the international multidisciplinary classification of the idiopathic interstitial pneumonias. Am J Respir Crit Care Med 2013; 188: 733-748.

4 Collins BF, Spiekerman CF, Shaw MA, et al. Idiopathic interstitial pneumonia associated with autoantibodies: a large case series followed over 1 year. Chest 2017; 152: 103-112.

5 Kinder BW, Collard HR, Koth L, et al. Idiopathic nonspecific interstitial pneumonia: lung manifestation of undifferentiated connective tissue disease? Am J Respir Crit Care Med 2007; 176: 691-697.

6 Corte TJ, Copley SJ, Desai SR, et al. Significance of connective tissue disease features in idiopathic interstitial pneumonia. Eur Respir J 2012; 39: 661-668.

7 Fischer A, West SG, Swigris JJ, Brown KK, du Bois RM. Connective tissue disease-associated interstitial lung disease: a call for clarification. Chest 2010; inflamed mucosa. ${ }^{(49)}$ Enhancement of Th1/Th17 inflammation by inhibition of Ro52 was also proposed to have a role in Beçhet disease. ${ }^{(50)}$ Nothing has been reported about lung inflammation in Ro52 knockout mice or humans. 1, 25-dihydroxycholecalciferol calcitriol, $\left[1,25(\mathrm{OH})_{2} \mathrm{D}_{3}\right]$ is activate form of vitamin $\mathrm{D}$, and calcitriol inhibited IL-23/IL-17 in the airway of cystic fibrosis patients. ${ }^{(51)}$ Furthermore, calcitriol prevented experimental lung fibrosis model in mice via inhibiting inflammation and accumulation of activated fibroblasts. ${ }^{(52)}$ Thus, IL-23/IL-17 pathway possibly is involved in IP development, however our preliminary examination of the serum IL-17 concentration did not show apparent elevation of IL-17A in Ro52 positive patients. To further investigate the contribution of Th17-based inflammation to IP, it would be necessary to examine lung tissue specimens.

There were several limitations of the present study. Treatment for IP depended on the attending doctors, although corticosteroids and/or immunosuppressive agents were used to treat all of the patients with lung-related death.

In conclusion, Ro52 positive IP patients without CTDs had worse 2-year survival than Ro52 negative patients, even though most of them showed a low ANA titer. Therefore, these patients require careful observation to detect progression of IP and allow initiation of medication with appropriate timing.

\section{Author Contributions}

HM undertook data collection, contributed to discussion the results and wrote the manuscript. YS designed the study, undertook data collection, contributed to discussion the results and wrote the manuscript. KK designed the study and contributed to discussion the results. YN, HO, TW, TY, SS, TS, KC undertook data collection and treat patients. NI, MM, and AT determine the CT patterns.

\section{Acknowledgments}

The authors are grateful to Yukiko Horigane, Mio Fujimaki, Reiko Komura (Department of Respiratory and Immunology), Keiko Hatanaka and Ayae Tanaka (Department of Rheumatology) for assistance with measuring serum.

\section{Conflict of Interest}

No potential conflicts of interest were disclosed.
138: $251-256$.

8 Vij R, Noth I, Strek ME. Autoimmune-featured interstitial lung disease: a distinct entity. Chest 2011; 140: 1292-1299.

9 Fischer A, Antoniou KM, Brown KK, et al.; ERS/ATS Task Force on Undifferentiated Forms of CTD-ILD. An official European Respiratory Society/American Thoracic Society research statement: interstitial pneumonia with autoimmune features. Eur Respir J 2015; 46: 976-987.

10 Alsumrain M, De Giacomi F, Mirza S, Moua T. Utility of autoimmune serology testing in the assessment of uncharacterized interstitial lung disease: a large retrospective cohort review. Respir Res 2017; 18: 161.

11 Mathews MB, Bernstein RM. Myositis autoantibody inhibits histidyl-tRNA synthetase: a model for autoimmunity. Nature 1983; 304: 177-179.

12 Nakashima R, Imura Y, Kobayashi S, et al. The RIG-I-like receptor IFIH1/ MDA5 is a dermatomyositis-specific autoantigen identified by the antiCADM-140 antibody. Rheumatology (Oxford) 2010; 49: 433-440.

13 Ghirardello A, Borella E, Beggio M, Franceschini F, Fredi M, Doria A. Myositis autoantibodies and clinical phenotypes. Auto Immun Highlights 2014; 5: 69-75.

14 Karakus S, Baer AN, Agrawal D, Guraker M, Massof RW, Akpek EK. Utility of novel autoantibodies in the diagnosis of Sjögren's syndrome among patients with dry eye. Cornea 2018; 37: 405-411. 
15 Rönnelid J, Barbasso Helmers S, Storfors H, et al. Use of a commercial line blot assay as a screening test for autoantibodies in inflammatory myopathies. Autoimmun Rev 2009; 9: 58-61.

16 Ghirardello A, Rampudda M, Ekholm L, et al. Diagnostic performance and validation of autoantibody testing in myositis by a commercial line blot assay. Rheumatology (Oxford) 2010; 49: 2370-2374.

17 Raghu G, Remy-Jardin M, Myers JL, et al.; American Thoracic Society, European Respiratory Society, Japanese Respiratory Society, and Latin American Thoracic Society. Diagnosis of idiopathic pulmonary fibrosis. An official ATS/ERS/JRS/ALAT clinical practice guideline. Am J Respir Crit Care Med 2018; 198: e44-e68.

18 Pan JA, Sun Y, Jiang YP, et al. TRIM21 ubiquitylates SQSTM1/p62 and suppresses protein sequestration to regulate redox homeostasis. Mol Cell 2016; 61: 720-733.

19 Shimizu Y, Dobashi K, Nagase H, et al. Co-localization of iron binding on silica with p62/sequestosome1 (SQSTM1) in lung granulomas of mice with acute silicosis. J Clin Biochem Nutr 2015; 56: 74-83.

20 Bohan A, Peter JB. Polymyositis and dermatomyositis (second of two parts). N Engl J Med 1975; 292: 403-407.

21 Kang EH, Lee EB, Shin KC, et al. Interstitial lung disease in patients with polymyositis, dermatomyositis and amyopathic dermatomyositis. Rheumatology (Oxford) 2005; 44: 1282-1286.

22 Aletaha D, Neogi T, Silman AJ, et al. 2010 rheumatoid arthritis classification criteria: an American College of Rheumatology/European League Against Rheumatism collaborative initiative. Ann Rheum Dis 2010; 69: 1580-1588.

23 van den Hoogen F, Khanna D, Fransen J, et al. 2013 classification criteria for systemic sclerosis: an American College of Rheumatology/European League against Rheumatism collaborative initiative. Arthritis Rheum 2013; 65: 2737 2747.

24 Jennette JC, Falk RJ, Bacon PA, et al. 2012 revised International Chapel Hill Consensus Conference Nomenclature of Vasculitides. Arthritis Rheum 2013; 65: $1-11$.

25 Kurasawa K, Arai S, Namiki Y, et al. Tofacitinib for refractory interstitial lung diseases in anti-melanoma differentiation-associated 5 gene antibodypositive dermatomyositis. Rheumatology (Oxford) 2018; 57: 2114-2119.

26 Fujibayashi T, Sugai S, Miyasaka N, et al. Revised Japanese criteria for Sjögren's syndrome. In: Annual Report of the Ministry of Health and Welfare, Autoimmune Disease Research Committee 1998, 1999; 135-138. (in Japanese)

27 Fujibayashi T, Sugai S, Miyasaka N, Hayashi Y, Tsubota K. Revised Japanese criteria for Sjögren's syndrome (1999): availability and validity. Mod Rheumatol 2004; 14: 425-434.

28 Lederer DJ, Martinez FJ. Idiopathic pulmonary fibrosis. N Engl J Med 2018; 379: 797-798.

29 Duchemann B, Annesi-Maesano I, Jacobe de Naurois C, et al. Prevalence and incidence of interstitial lung diseases in a multi-ethnic county of Greater Paris. Eur Respir J 2017; 50: 1602419.

30 Song JS, Hwang J, Cha HS, et al. Significance of myositis autoantibody in patients with idiopathic interstitial lung disease. Yonsei Med J 2015; 56: 676683.

31 Yamasaki Y, Satoh M, Mizushima M, et al. Clinical subsets associated with different anti-aminoacyl transfer RNA synthetase antibodies and their association with coexisting anti-Ro52. Mod Rheumatol 2016; 26: 403-409.

32 Wodkowski M, Hudson M, Proudman S, et al. Monospecific anti-Ro52/ TRIM21 antibodies in a tri-nation cohort of 1574 systemic sclerosis subjects: evidence of an association with interstitial lung disease and worse survival. Clin Exp Rheumatol 2015; 33: S131-S135.

33 Gunnarsson R, El-Hage F, Aaløkken TM, et al. Associations between antiRo52 antibodies and lung fibrosis in mixed connective tissue diseaseRheumatology (Oxford) 2016; 55: 103-108.

34 Hudson M, Pope J, Mahler M, et al. Clinical significance of antibodies to Ro52/TRIM21 in systemic sclerosis. Arthritis Res Ther 2012; 14: R50.
35 Murng SHK, Thomas M. Clinical associations of the positive anti Ro52 without Ro60 autoantibodies: undifferentiated connective tissue diseases. $J$ Clin Pathol 2018; 71: 12-19.

36 Yoshimi R, Ueda A, Ozato K, Ishigatsubo Y. Clinical and pathological roles of Ro/SSA autoantibody system. Clin Dev Immunol 2012; 2012: 606195.

37 Espinosa A, Dardalhon V, Brauner S, et al. Loss of the lupus autoantigen Ro52/Trim 21 induces tissue inflammation and systemic autoimmunity by disregulating the IL-23-Th17 pathway. J Exp Med 2009; 206: 1661-1671.

38 Grasselli G, Vergnano B, Pozzi MR, et al. Interstitial pneumonia with autoimmune features: an additional risk factor for ARDS? Ann Intensive Care 2017; 7: 98 .

39 Travis WD, Hunninghake G, King TE Jr, et al. Idiopathic nonspecific interstitial pneumonia: report of an American Thoracic Society project. Am J Respir Crit Care Med 2008; 177: 1338-1347.

40 Srivastava P, Dwivedi S, Misra R. Myositis-specific and myositis-associated autoantibodies in Indian patients with inflammatory myositis. Rheumatol Int 2016; 36: 935-943.

41 Hussain A, Rawat A, Jindal AK, Gupta A, Singh S. Autoantibodies in children with juvenile dermatomyositis: a single centre experience from North-West India. Rheumatol Int 2017; 37: 807-812.

42 Takada T, Hirakata M, Suwa A, et al. Clinical and histopathological features of myopathies in Japanese patients with anti-SRP autoantibodies. Mod Rheumatol 2009; 19: 156-164.

43 Hanaoka H, Kaneko Y, Suzuki S, et al. A unique case of polymyositis with anti-signal recognition particle antibody complicated by subacute interstitial lung disease and subluxing arthropathy, resembling anti-synthetase syndrome. Mod Rheumatol 2016; 26: 979-980.

44 Kusumoto T, Okamori S, Masuzawa K, et al. Development of necrotizing myopathy following interstitial lung disease with anti-signal recognition particle antibody. Intern Med 2018; 57: 2045-2049.

45 Espinosa A, Hennig J, Ambrosi A, et al. Anti-Ro52 autoantibodies from patients with Sjögren's syndrome inhibit the Ro52 E3 ligase activity by blocking the E3/E2 interface. J Biol Chem 2011; 286: 36478-36491.

46 Rajsbaum R, García-Sastre A, Versteeg GA. TRIMmunity: the roles of the TRIM E3-ubiquitin ligase family in innate antiviral immunity. $J$ Mol Biol 2014; 426: 1265-1284.

47 Rhodes DA, Trowsdale J. TRIM21 is a trimeric protein that binds IgG Fc via the B30.2 domain. Mol Immunol 2007; 44: 2406-2414.

48 Dickson C, Fletcher AJ, Vaysburd M, et al. Intracellular antibody signalling is regulated by phosphorylation of the Fc receptor TRIM21. Elife 2018; 7. pii: e32660.

49 Zhou G, Wu W, Yu L, et al. Tripartite motif-containing (TRIM) 21 negatively regulates intestinal mucosal inflammation through inhibiting $\mathrm{T}_{\mathrm{H}} 1 / \mathrm{T}_{\mathrm{H}} 17$ cell differentiation in patients with inflammatory bowel diseases. $J$ Allergy Clin Immunol 2018; 142: 1218-1228.e12.

50 Ahn Y, Hwang JH, Zheng Z, Bang D, Kim DY. Enhancement of Th1/Th17 inflammation by TRIM21 in Behçet's disease. Sci Rep 2017; 7: 3018.

51 Olszowiec-Chlebna M, Koniarek-Maniecka A, Brzozowska A, Błauż A, Rychlik B, Stelmach I. Vitamin D inhibits pro-inflammatory cytokines in the airways of cystic fibrosis patients infected by Pseudomonas aeruginosa- pilot study. Ital J Pediatr 2019; 45: 41.

52 Tsujino I, Ushikoshi-Nakayama R, Yamazaki T, Matsumoto N, Saito I. Pulmonary activation of vitamin $\mathrm{D}_{3}$ and preventive effect against interstitial pneumonia. J Clin Biochem Nutr 2019; 65: 245-251.

This is an open access article distributed under the terms of the Creative Commons Attribution License, which permits unrestricted use, distribution, and reproduction in any medium, provided the original work is properly cited. 